\title{
Магнитозвуковые волны в двумерной электронной ферми-жидкости
}

\author{
(C) П.С. Алексеев \\ Физико-технический институт им. А.Ф. Иоффре Российской академии наук \\ 194021 Санкт-Петербург, Россия \\ E-mail: pavel.alekseev@mail.ioffe.ru
}

Поступила в Редакцию 22 мая 2019 г.

В окончательной редакции 23 мая 2019 г.

Принята к публикации 29 мая 2019 г.

\begin{abstract}
Свойства сильновязких жидкостей при высоких частотах становятся похожи на свойства аморфных твердых тел. В частности, становится возможно распространение не только продольных звуковых волн (плазмонов для случая электронной жидкости), но также и поперечных звуковых волн, связанных со сдвиговыми деформациями. В работе изучено возникновение поперечных волн при высоких частотах в двумерной электронной жидкости в магнитном поле. Рассмотрение проведено в рамках модели фермижидкости Ландау. Показано, что при достаточно большой силе взаимодействия квазичастиц динамика возбуждений ферми-жидкости описывается уравнениями гидродинамики. Выведено уравнение Навье-Стокса и выражения для высокочастотных коэффициентов сдвиговой вязкости. На основе полученных уравнений рассчитаны законы дисперсии для поперечных и продольных магнитозвуковых волн. Показано, что циклотронная частота, входящая в коэффициенты вязкости и закон дисперсии поперечных магнетозвуковых волн, перенормируется и типично становится меньше обычной циклотронной частоты, определяющей циклотронный резонанс. Последний факт, по-видимому, наблюдался в фотосопротивлении высокоподвижных квантовых ям GaAs, в которых двумерные электроны формируют вязкую жидкость.
\end{abstract}

Ключевые слова: электронная ферми-жидкость, вязкость, магнетотранспорт, магнитный резонанс.

DOI: 10.21883/FTP.2019.10.48298.9166

\section{1. Введение}

В достаточно чистых кристаллических материалах фононы и электроны проводимости при низких температурах могут формировать вязкую жидкость при условии, что сохраняющие импульс межчастичные столкновения являются значительно более интенсивными, чем другие типы столкновений, не сохраняющие импульс [1]. Фононный гидродинамический транспорт в жидком гелии, диэлектриках, металлах и полупроводниках был достаточно детально теоретически и экспериментально изучен несколько десятилетий назад [2-4]. Однако гидродинамический режим электронного транспорта в проводниках был экспериментально открыт только недавно в новых ультрачистых материалах: квантовых ямах GaAs [5-10], моновалентном слоистом металле $\mathrm{PdCoO}_{2}$ [11], объемном вейлевском металле $\mathrm{WP}_{2}$ [12] и графене [13-15]. Эти экспериметальные открытия сопровождались интенсивным развитием теории [16-31].

Одним из доказательств наличия гидродинамического режима электронного транспорта является эффект гигантского отрицательного магнитосопротивления, котороый состоит в уменьшении сопротивления образца на 1-2 порядка в умеренных магнитных полях. Такое магнетосопротивление было недавно обнаружено в квантовых ямах GaAs самого высокого качества [5-8], вейлевском полуметалле $\mathrm{WP}_{2}$ [12] и графене [15]. Этот эффект был объяснен с помощью гидродинамической модели, учитывающей зависимость коэффициентов вязкости электронов от магнитного поля и температуры [18]. Другим доказательством формирования вязкой электронной жидкости в графеновых и высокоподвижных квантовых ямax $\mathrm{GaAs}$ является наблюдение отрицательного нелокального сопротивления, связанного с образованием водоворотов в электронной жидкости [10-19].

Высокочастотный транспорт в вязкой электронной жидкости теоретически рассматривался в нескольких недавних публикациях [27-32]. Переменное течение жидкости в длинном образце в нулевом магнитном поле изучалось в рамках гидродинамического подхода в [27,28]. В работе [30] были рассмотрены поперечный нулевой звук и плазмоны в двумерной сильно неидеальной ферми-жидкости в отсутствие магнитного поля. В статье [31] было показано, что коэффициенты вязкости двумерных электронов имеют резонанс на частоте $\omega$, равной удвоенной циклотронной частоте электронов $2 \omega_{c}$.

В работе [32] в рамках феноменологических уравнений гидродинамики было предсказано, что в сильно вязкой электронной жидкости при достаточно высоких частотах могут возбуждаться поперечные магнитозвуковые волны. Такие волны аналогичны поперечному звуку в аморфных твердых телах, иными словами, их появление с ростом частоты означает вязкоупругий переход в системе. Было также показано, что в образце малого размера предсказанные волны приводят к асимметричному резонансу в отклике жидкости на радиочастотное поле при $\omega=2 \omega_{c}$, что, по-видимому, наблюдалось в высокоподвижных квантовых ямах $\mathrm{GaAs}$ в экспериментах [33-35]. В работе [32] было установлено, что необходимым условием распространения поперечных магнитозвуковых волн является высокая 
степень ее неидеальности, выражающаяся в том, что коэффициенты вязкости жидкости много больше их значений для идеального ферми-газа ${ }^{1}$.

Настоящая работа посвящена построению микроскопической теории магнитозвуковых волн в системе двумерных электронов в рамках модели ферми-жидкости Ландау [38]. Показано, что кинетическое уравнение для квазичастиц ферми-жидкости переходит в гидродинамическое уравнение Навье-Стокса для случая сильного взаимодействия квазичастиц (большие амплитуда и анизотропия функции Ландау). Это подтверждает сделанное в работе [32] заключение о возможности гидродинамического рассмотрения поперечных магнитозвуковых волн в сильнонеидеальной жидкости. Рассчитаны законы дисперсии поперечного и продольного нулевого звука в магнитном поле. Показано, что циклотронная частота, входящая в коэффициенты вязкости и закон дисперсии поперечного звука, переномируется: при наиболее вероятном соотношении между гармониками функции Ланаду такая циклотронная частота уменьшается по сравнению с циклотронной частой невзаимодействующих электронов, определяющей циклотронный резонанс ферми-жидкости. Этот результат представляется вероятным объяснением того, что в экспериментах [33-35] резонанс в ряде случаев наблюдался на частоте меньше, чем удвоенное экспериментальное значение частоты циклотронного резонанса.

\section{2. Кинетическое уравнение и потоковые величины}

Из-за межэлектронного взаимодействия электроны проводимости в двумерных проводниках, вообще говоря, представляют собой ферми-жидкость, хотя часто в реальных системах межэлектронное взаимодействие можно не учитывать. Состояние заряженной фермижидкости определяется квазичастицами похожими на электроны и дырки вырожденного ферми-газа, находящиеся над ферми-поверхностью и под ней $[39,40]$. Одним из способов описания таких частиц является использование функции распределения „квазичастиц в заполненных состояниях“ типа функции распределения частиц ферми-газа.

В случае слабого отклонения от равновесия функция распределения квазичастиц может быть представлена в виде

$$
f_{\mathbf{p}}=f_{\mathrm{F}}\left(\varepsilon_{p}^{0}\right)+\delta f_{\mathbf{p}},
$$

где $f_{\mathrm{F}}(E)=1 /\left[1+e^{(E-\mu) / T}\right]-$ фермиевская функция, $\delta f_{\mathbf{p}}$ - малая поправка к ней, $T-$ равновесная температура, $\mu$ - равновесный химический потенциал, $\varepsilon_{p}^{0}=p^{2} /(2 m)-$ энергетический спектр квазичастицы

\footnotetext{
1 Отметим, что для высокоподвижных квантовых ям GaAs имеются экспериментальные свидетельства того, что такое условие сильной неидеальности электронной жидкости действительно может быть выполнено [36,37]
}

при отсутсвии других квазичастиц. По смыслу квазичастиц как низковозбужденных состояний жидкости $f_{\mathbf{p}}$ определена только при импульсах $\mathbf{p}$, лежащих около поверхности Ферми $|\mathbf{p}|=p_{\mathrm{F}}$. Соотношением $\mathbf{v}_{\mathbf{p}}=\partial \varepsilon_{p}^{0} / \partial \mathbf{p}$ определяется скорость квазичастицы при отсутсвии других квазичастиц. Отметим, что в формуле $(1) f_{\mathrm{F}}\left(\varepsilon_{p}^{0}\right)$ не есть равновесная часть функции распределения $f_{\mathbf{p}}$, так как $\varepsilon_{p}^{0}$ отвечает отсутствию квазичастиц.

Ключевым в теории ферми-жидкости является учет зависимости энергии квазичастиц от функции распределения квазичастиц:

$$
\varepsilon_{\mathbf{p}}=\varepsilon_{p}^{0}+\sum_{\mathbf{p}^{\prime}} f_{\mathbf{p p}^{\prime}} \delta f_{\mathbf{p}^{\prime}},
$$

где $f_{\mathbf{p p}^{\prime}}$ - функция Ландау, описывающая взаимодействие квазичастиц. В формуле (2) опущены спиновые переменные, так как в настоящей статье не будут изучаться спин-зависмые явления. В случае зависимости $\delta f_{\mathbf{p}}$ от координат и от времени энергия квазичастиц в силу (2) также становится нелокальной по времени и пространству. Учету взаимодействия квазичастиц согласно (2), соответствует возмущенная скорость квазичастиц $\tilde{\mathbf{v}}_{\mathbf{p}}=\partial \varepsilon_{\mathbf{p}} / \partial \mathbf{p}$. Равновесная часть функции распределения $f_{\mathbf{p}}$ вида (1) приобретает вид $f_{\mathrm{F}}\left(\varepsilon_{\mathbf{p}}\right)$, поэтому можно найти неравновесную часть функции $f_{\mathbf{p}}$ по формуле

$$
\delta \tilde{f}_{\mathbf{p}}=f_{\mathbf{p}}-f_{\mathrm{F}}\left(\varepsilon_{\mathbf{p}}\right) .
$$

Заметим, что возмущение скорости квазичастиц за счет взаимодействия мало $\left|\tilde{\mathbf{v}}_{\mathbf{p}}-\mathbf{v}_{\mathbf{p}}\right| \ll v_{\mathrm{F}} \quad\left(v_{\mathrm{F}}=p_{\mathrm{F}} / m\right)$, но поправка к функции распределения $\delta f_{\mathbf{p}}$, определенная без учета взаимодейстивя, и истинная неравновесная поправкая $\delta \tilde{f}_{\mathbf{p}}$ - величины одного порядка:

$$
\left|\delta f_{\mathbf{p}}-\delta \tilde{f}_{\mathbf{p}}\right| \sim\left|\delta f_{\mathbf{p}}\right| .
$$

Связь между ними дается формулой

$$
\delta \tilde{f}_{\mathbf{p}}=\delta f_{\mathbf{p}}-f_{\mathrm{F}}^{\prime}\left(\varepsilon_{p}^{0}\right) \sum_{\mathbf{p}^{\prime}} f_{\mathbf{p p}^{\prime}} \delta f_{\mathbf{p}^{\prime}} .
$$

Динамика заряженных квазичастиц во внешних электрическом и магнитном полях $\mathbf{E}_{0}$ и $\mathbf{B}_{0}$ при условии, что не важны эффекты электромагнитного запаздывания, описывается кинетическим уравнением $[40,41]$

$$
\begin{aligned}
\frac{\partial \delta f_{\mathbf{p}}}{\partial t} & +\mathbf{v}_{\mathbf{p}} \frac{\partial \delta \tilde{f}_{\mathbf{p}}}{\partial \mathbf{r}}+\frac{e}{c}\left[\mathbf{v}_{\mathbf{p}} \times \mathbf{B}_{0}\right] \\
& \times \frac{\partial \delta \tilde{f}_{\mathbf{p}}}{\partial \mathbf{p}}+e \mathbf{E} \cdot \mathbf{v}_{\mathbf{p}} f_{\mathrm{F}}^{\prime}\left(\varepsilon_{\mathbf{p}}^{0}\right)=\operatorname{St}\left[\delta \tilde{f}_{\mathbf{p}}\right],
\end{aligned}
$$

где электрическое поле состоит из внешнего поля $\mathbf{E}_{0}$ и внутреннего „власовского“ квазистатического поля, связанного с возмущением плотности квазичастиц соотношениями электростатики. Для случая двумерных электронов в системе с затвором имеем

$$
\mathbf{E}=\mathbf{E}_{0}-\frac{4 \pi d e}{\kappa} \frac{\partial \delta n}{\partial \mathbf{r}}, \quad \delta n=\sum_{\mathbf{p}^{\prime}} \delta f_{\mathbf{p}^{\prime}} .
$$


В последнюю формулу входит именно $\delta f_{\mathbf{p}}$, а не $\delta \tilde{f}_{\mathbf{p}}=f_{\mathbf{p}}-f_{\mathrm{F}}\left(\varepsilon_{\mathbf{p}}\right)$, так как в силу (2) величина $f_{\mathrm{F}}\left(\varepsilon_{\mathbf{p}}\right)$ зависит от координаты и дает свой вклад во внутреннее электрическое поле. В формулах (6) и (7) учитывается, что квазичастицы имеют заряд $e$, как и исходные электроны. Напомним, что величина

$$
n_{0}=\sum_{\mathbf{p}} f_{\mathbf{p}}-\delta n
$$

не имеет прямого физического смысла, но при нуле температур равна концентрации исходных электронов (гипотеза Ландау, доказанная в рамках обоснования теории ферми-жидкости методами квантовой теории поля [39]). Важно, что в уравнении (6) в динамические слагаемые с градиентом функции распределения, с внешним магнитным полем и в интеграл столкновений за счет межчастичных столкновений $\operatorname{St}\left[\delta \tilde{f}_{\mathbf{p}}\right]$ входит неравновесная часть функции распреления $\delta \tilde{f}_{\mathbf{p}}$, а в слагаемые с производной по времени и с внутренним электрическим полем входит неоднородная в пространстве часть функции распределения $\delta f_{\mathbf{p}}$. Уравнение (6) описывает эффекты в области образца, где возмущения величин: температуры, потока и плотности частиц линейны по какому-либо малому внешнему параметру, например по внешнему полю $\mathbf{E}_{0}$. По последней причине в (6) всюду входит скорость без учета взаимодействия $\mathbf{v}_{\mathbf{p}}$. Напомним, что интеграл St сохраняет число квазичастиц, импульс и энергию, но не сохраняет плотность потока импульса.

Выражение для плотности потока частиц выводится из интегрирования (6) по импульсам и выделения из полученного уравнения непрерывности величин, соответствующих плотности потока. В результате получается

$$
\mathbf{j}=\sum_{\mathbf{p}} \mathbf{v}_{\mathbf{p}} \delta \tilde{f}_{\mathbf{p}}
$$

Выражение для плотности потока импульса частиц получается аналогичным образом: умножением (6) на $p_{i}$, интегрированием по импульсам, и выделением из полученного уравнения сохранения импульса соответствующих слагаемых:

$$
\Pi_{i j}=\sum_{\mathbf{P}} p_{i} v_{\mathbf{p} j} \delta \tilde{f}_{\mathbf{p}}
$$

Эта величина может быть разделена на часть, связанную с возмущением всестороннего сжатия (т.е. давления $P$ ), и на часть, связанную с вязкими напряжениями: $\Pi_{i j}=\Pi_{i j}^{\delta P}+\Pi_{i j}^{\prime}$. Первая из них определяется выражением

$$
\Pi_{i j}^{\delta P}=\frac{1}{2} \delta_{i j} \sum_{\mathbf{p}} p v_{p} \delta \tilde{f}_{\mathbf{p}} .
$$

Видно, что эта величина пропорциональна плотности энергии квазичастиц:

$$
\delta \epsilon=\sum_{\mathbf{p}} \varepsilon_{\mathbf{p}}^{0} \delta \tilde{f}_{\mathbf{p}} \approx \sum_{\mathbf{p}} \varepsilon_{\mathbf{p}} \delta \tilde{f}_{\mathbf{p}} .
$$

Вязкостная часть плотности потока импульса приобретает вид

$$
\Pi_{i j}^{\prime}=\sum_{\mathbf{p}}\left[p_{i} v_{\mathbf{p} j}-\delta_{i j} \frac{p v_{p}}{2}\right] \delta \tilde{f}_{\mathbf{p}}
$$

Для вывода уравнений гидродинамики необходимо ввести также величину, описывающую поток плотности потока импульса:

$$
\Pi_{i j k}=\sum_{\mathbf{p}} p_{i} v_{\mathbf{p} j} v_{\mathbf{p} k} \delta \tilde{f}_{\mathbf{p}}
$$

Подобно плотности потока импульса, введенная величина содержит две части разной природы:

$$
\Pi_{i j k}=\Pi_{i j k}^{J}+\Pi_{i j k}^{\prime \prime} .
$$

Вторая из них, $\Pi_{i j k}^{\prime \prime}$, пропорциональна третьей угловой гармонике функции распределения, которая в гидродинамическом режиме считается пренебрежимо малой. Слагаемое $\Pi_{i j k}^{J}$, подобное части $\Pi_{i j}^{\delta P}$ плотности потока импульса, пропорционально первым гармоникам функции распределения, обозначаемым $\left(\delta \tilde{f}_{\mathbf{p}}\right)_{m=1}$

$$
\Pi_{i j k}^{J}=\sum_{\mathbf{p}} p_{i} v_{\mathbf{p} j} v_{\mathbf{p} k}\left(\delta \tilde{f}_{\mathbf{p}}\right)_{m=1}
$$

т. е. потоку квазичастиц ј. В следующем разделе будет установлена конкретная свзь между компонентами $\Pi_{i j k}^{J}$ и $j_{i}$.

\section{3. Уравнение для волн}

Решения кинетического уравнения будем изучать в приближении простейшей зависимости функций распределения от энергии:

$$
\delta f_{\mathbf{p}}=-f_{\mathrm{F}}^{\prime}\left(\varepsilon_{p}^{0}\right) \Phi(\varphi), \quad \delta \tilde{f}_{\mathbf{p}}=-f_{\mathrm{F}}^{\prime}\left(\varepsilon_{p}^{0}\right) \tilde{\Phi}(\varphi),
$$

где $\varphi$ - полярный угол вектора р. Неучет в функциях $\Phi$ и $\tilde{\Phi}$ зависмости от энергии означает, что мы не рассматриваем эффекты типа теплопроводности и объемной вязкости, связанные с изменением энергии квазичастиц при их рассеянии. Функции $\Phi$ и $\tilde{\Phi}$ такого вида описывают в нулевом приближении по $T / \mu$ эффекты, связанные с возмущеним плотности, с циклотронным действием магнитного поля и с изменением направления квазиимпульсов при рассеянии. Например, такими эффектами являются плазменные волны, звуковые волны и сдвиговая вязкость.

В связи с выбранным анзацем (15) используем функцию Ландау $f_{\mathbf{p p}}$, не зависящую от модулей импульсов и обычным образом обезразмеренную: $F\left(\varphi-\varphi^{\prime}\right)=\nu_{\mathrm{F}} f_{\mathbf{p p}^{\prime}}$, где $v_{\mathrm{F}}=m /\left(2 \pi \hbar^{2}\right)$ - плотность состояний. Получим следующую связь функций (15):

$$
\tilde{\Phi}(\varphi)=\Phi(\varphi)+\int \frac{d \varphi^{\prime}}{2 \pi} F\left(\varphi-\varphi^{\prime}\right) \Phi\left(\varphi^{\prime}\right)
$$


и следующую форму кинетического уравнения:

$$
\begin{aligned}
& \frac{\partial \Phi}{\partial t}+v_{\mathrm{F}}\left(\begin{array}{c}
\cos \varphi \\
\sin \varphi
\end{array}\right) \frac{\partial \tilde{\Phi}}{\partial \mathbf{r}}-\omega_{c} \frac{\partial \tilde{\Phi}}{\partial \varphi} \\
& +\frac{2 s^{2}}{v_{\mathrm{F}}}\left(\begin{array}{c}
\cos \varphi \\
\sin \varphi
\end{array}\right) \int \frac{d \varphi^{\prime}}{2 \pi} \frac{\partial \Phi\left(\varphi^{\prime}\right)}{\partial \mathbf{r}}-e \mathbf{E}_{0} v_{\mathrm{F}}\left(\begin{array}{c}
\cos \varphi \\
\sin \varphi
\end{array}\right)=\mathrm{St}[\tilde{\Phi}],
\end{aligned}
$$

где

$$
s^{2}=\frac{4 \pi e^{2} d}{\kappa} \frac{v_{\mathrm{F}}^{2}}{2} v_{\mathrm{F}}=\frac{4 \pi e^{2} d n_{0}}{m \kappa}
$$

- квадрат скорости плазмона в отсутствие магнитного поля и взаимодействия. Мы считаем, что, как обычно, $s \gg v_{\mathrm{F}}$. Величина $\mathrm{St}[\tilde{\Phi}]$ представляет собой среднее по модулю импульса $p=|\mathbf{p}|$ от $\operatorname{St}\left[\delta \tilde{f}_{\mathbf{p}^{\prime}}\right]_{\mathbf{p}}$. При этом игнорируется различие в зависимости от $p$ для левой и правой частей кинетическокого уравнения при подстановке в него $\delta \tilde{f}_{\mathbf{p}^{\prime}}$ вида (15). Это приближение дает ошибку, пропорциональную $T / \varepsilon_{\mathrm{F}}$, и соответствует использованию анзаца (15).

С помощью полученного уравнения (16) можно, вопервых изучить волны, возможные в системе. Выберем функции распределения в виде

$$
\Phi(\varphi, \mathbf{r}, t)=A(\varphi) e^{-i \omega t+i q x}, \quad \tilde{\Phi}(\varphi, \mathbf{r}, t)=\tilde{A}(\varphi) e^{-i \omega t+i q x} .
$$

Без учета процессов релаксации система уравнений для амплитуд волн приобретает вид

$$
\left\{\begin{array}{c}
-i \omega A(\varphi)+v_{\mathrm{F}} \cos \varphi i q \tilde{A}(\varphi)-\omega_{c} \frac{d \tilde{A}}{d \varphi} \\
+\frac{2 s^{2}}{v_{\mathrm{F}}} i q \int \frac{d \varphi^{\prime}}{2 \pi} A\left(\varphi^{\prime}\right)=0, \\
\tilde{A}(\varphi)=A(\varphi)+\int \frac{d \varphi^{\prime}}{2 \pi} F\left(\varphi-\varphi^{\prime}\right) A\left(\varphi^{\prime}\right) .
\end{array}\right.
$$

Эти уравнения являются задачей на собственные функции $A(\varphi)$ и собственные значения $\omega$ с интегродифференциальным оператором. Из полученной ситемы, например, сразу легко показать, что для простейшего случая, когда отсутствует взаимодействие $(F=0)$ и рассматриваются большие длины волн $\left(q v_{\mathrm{F}} \ll \omega\right.$ или $\left.q v_{\mathrm{F}} \ll \omega_{c}\right)$, получается обычный спектр магнетоплазмонов: $\omega_{q}=\sqrt{\omega_{c}^{2}+v_{\mathrm{F}}^{2} q^{2}}$.

Полученная система уравнений (18) есть обобщение приведенных в книгах $[39,40]$ уравнений, описывающих нулевой звук в заряженной ферми-жидкости, на случай наличия магнитного поля. Уравнения (18) позволяют, вопервых, изучить влияние ферми-жидкостных эффектов на плазменные волны, и, во-вторых, изучить остальные типы волн нулевого звука при произвольных амплитуде и профиле функции Ландау $F(\varphi)$ и при произвольных длинах волн и соотношениях между частотами $\omega$ и $\omega_{c}$.

Далее в настоящей работе будет показано, что при достаточно большой по абсолютной величине и амплитуде изменения $F(\varphi)$ кинетическое уравнение (16) сводится к уравнениям гидродинамики. На основе последних будут изучены поперечные и продольные магнитозвуковые волны в длинноволновом пределе. Задача изучения магнитозвуковых и магнетоплазменных волн при произвольной силе взаимодействия с помощью (18) может быть актуальной, в частности, для количественного анализа экспериментальных данных, в зависимости от того, насколько окажется велика $F(\varphi)$ в реальных образцах, где происходят обсуждаемые явления.

\section{4. Уравнения гидродинамики}

Выведем уравнения гидродинамики заряженной ферми-жидкости в магнитном поле из кинетического уравнения $(16)^{2}$.

Назложим на угловые моменты функцию Ландау и функции распределения:

$$
\left(\begin{array}{c}
F(\varphi) \\
\Phi(\varphi) \\
\tilde{\Phi}(\varphi)
\end{array}\right)=\sum_{m=-\infty}^{\infty}\left(\begin{array}{c}
F_{m} \\
\Phi_{m} \\
\tilde{\Phi}_{m}
\end{array}\right) e^{i m \varphi}
$$

Параметры $F_{m}$ вещественны и $F_{m}=F_{-m}$ в силу симметричности функции Ландау при изменении $\varphi$ на $-\varphi$. Для сокращения записи дальнейших формул введенные выше величины $F_{m}$ отличаются от обычно используемых параметров разложения на множитель 2. Связь между $\Phi$ и $\widetilde{\Phi}$ выразится соотношением

$$
\tilde{\Phi}_{m}=\left(1+F_{m}\right) \Phi_{m}
$$

Гидродинамическое приближение работает в ситуации, когда форма потока является достаточно плавной, а именно: характерная длина волны много больше одной их внутренних характерных длин: $\omega / v_{\mathrm{F}}, \omega_{c} / v_{\mathrm{F}}$ либо длины релаксации. Как известно, такому течению соответствует малость гармоник функции распределения, ставших второй, по сравнению с гармониками $\Phi_{0}, \Phi_{ \pm 1}, \Phi_{ \pm 2}$. Из кинетического уравнения (16) и соотношений (20) можно получить критерий на параметры функции Ландау, когда это происходит (при условии, что характерная длина волны опре- деляется внутренними свойствами жидкости, а не, например, размером образца). Анализ показывает, что необходимы следующие неравенства:

$$
1+F_{m} \ll F_{1}, \sqrt{F_{1}\left(1+F_{1}\right)}
$$

при всех $m \geq 3$. При выполнении неравенств (21) открывается возможность получить замнутое уравнение для младших гармоник, т. е. для пропорциональных им потоковых величин $\mathbf{j}$ и $\Pi_{i j}$ и возмущения концентрации $\delta n$.

Для вывода уравнений эволюции плотности квазичастиц, плотности потока и плотности потока импульса необходимы явные выражения для этих величин через

\footnotetext{
2 Уравнения гидродинамики ферми-жидкости без магнитного поля впервые были получены в работе [42] и затем более подробно изучены в работе [43].
} 
функции распределения (15) в нулевом порядке по $T / \varepsilon_{\mathrm{F}}$. Для возмущения плотности и плотности потока получаем

$$
\begin{gathered}
\delta n=v_{\mathrm{F}} \int \frac{d \varphi}{2 \pi} \Phi(\varphi), \\
\mathbf{j}=v_{\mathrm{F}} v_{\mathrm{F}} \int \frac{d \varphi}{2 \pi}\left(\begin{array}{c}
\cos \varphi \\
\sin \varphi
\end{array}\right) \tilde{\Phi}(\varphi) .
\end{gathered}
$$

Для части плотности потока импульса, связанной с неоднородностью давления, имеем

$$
\Pi_{i j}^{\delta P}=v_{\mathrm{F}} \frac{m v_{\mathrm{F}}^{2}}{2} \int \frac{d \varphi}{2 \pi} \tilde{\Phi}(\varphi),
$$

что можно записать как

$$
\Pi_{i j}^{\delta P}=\frac{m v_{\mathrm{F}}^{2}}{2} \delta \tilde{n}, \quad \delta \tilde{n}=v_{\mathrm{F}} \int \frac{d \varphi}{2 \pi} \tilde{\Phi}(\varphi) .
$$

Для вязкостной части плотности потока импульса получается выражение

$$
\left(\begin{array}{l}
\Pi_{x x}^{\prime} \\
\Pi_{y y}^{\prime} \\
\Pi_{x y}^{\prime}
\end{array}\right)=v_{\mathrm{F}} m v_{\mathrm{F}}^{2} \int \frac{d \varphi}{2 \pi}\left(\begin{array}{c}
\cos ^{2} \varphi-1 / 2 \\
\sin ^{2} \varphi-1 / 2 \\
\sin \varphi \cos \varphi
\end{array}\right) \tilde{\Phi}(\varphi) .
$$

Видно, что последняя величина пропорциональна второй гармонике функции распределения: $\Pi_{i j}^{\prime} \sim \tilde{\Phi}_{m= \pm 2}$, в то время как часть от неоднородности давления пропорциональна нулевой гармонике, т. е. возмущению плотности: $\delta \tilde{n}=\left(1+F_{0}\right) \delta n$.

Как обсуждалось выше, одна из частей введенной величины $\Pi_{i j k}$, описывающая поток плотности потока импульса, пропорциональна третьей гармонике функции распределения: $\Pi_{i j k}^{\prime \prime} \sim \tilde{\Phi}_{m= \pm 3}$. Такая величина в гидродинамическом пределе, который реализуется при выполнении неравенств (21), является пренебрежимо малой. Для токовой части $\Pi_{i j k}$ из формул (9) и (14) можно получить соотношение

$$
\Pi_{i j k}^{j}=\frac{m v_{\mathrm{F}}^{2}}{4}\left(\delta_{i k} j_{j}+\delta_{i k} j_{i}-\delta_{i j} j_{k}\right) .
$$

Далее необходимо умножить кинетическое уравнение (16) последовательно на $1, v_{i}$ и $p_{i} v_{j}$, просуммировать по импульсам и преобразовать, использовав выражения для величин (22-26). Не приводя подробностей вычислений, приведем получающийся результат:

$$
\left\{\begin{array}{l}
\frac{\partial \delta n}{\partial t}+\frac{\partial j_{i}}{\partial x_{i}}=0 \\
\frac{1}{1+F_{1}} \frac{\partial j_{i}}{\partial t}+\frac{1}{m} \frac{\partial \Pi_{i j}}{\partial x_{j}}+s^{2} \frac{\partial \delta n}{\partial x_{i}}-\frac{e n_{0} E_{0 i}}{m}-\omega_{c} \epsilon_{i j z} j_{j}=0 \\
\frac{1}{1+F_{2}} \frac{\partial \Pi_{i j}^{\prime}}{\partial t}+\frac{\partial \Pi_{i j k}}{\partial x_{k}}-\omega_{c}\left(\epsilon_{j k z} \Pi_{i k}^{\prime}+\epsilon_{i k z} \Pi_{k j}^{\prime}\right)=-\frac{\Pi_{i j}^{\prime}}{\tau_{2}}
\end{array}\right.
$$

где $\epsilon_{i j l}-$ абсолютно антисимметричный тензор третьего ранга. В последнем уравнении слагаемое $-\Pi_{i j}^{\prime} / \tau_{2}$ описывает релаксацию второй гармоники функции распределения за счет $\mathrm{St}[\tilde{\Phi}]$. Гидродинамическое приближение состоит в замене в полученных уравнениях величины $\Pi_{i j k}$ на $\Pi_{i j k}^{J}$, что означает учет пренебрежимой малости третьей и старших гармоник функции распределения.

После этой замены, используя (25) и (27), последние два уравнения в (28) можно преобразовать к виду, похожему на вид аналогичных по смыслу уравнений для ферми-газа, полученных в работах $[18,31]$ :

$$
\left\{\begin{array}{l}
\frac{\partial j_{i}}{\partial t}+s_{0}^{2} \frac{\partial \delta n}{\partial x_{i}}-\frac{e n_{0} E_{0 i}}{m_{0}}-\omega_{c}^{(0)} \epsilon_{i j z} j_{j} \\
\quad+\left(1+F_{1}\right)\left(1+F_{0}\right) \frac{v_{\mathrm{F}}^{2}}{2} \frac{\partial \delta n}{\partial x_{i}}+\frac{1+F_{1}}{m} \frac{\partial \Pi_{i j}^{\prime}}{\partial x_{j}}=0 \\
\quad+\left(1+F_{2}\right) \frac{m \eta_{\mathrm{F}}^{\prime}}{4}\left[\frac{\partial j_{i}}{\partial x_{j}}+\frac{\partial j_{j}}{\partial x_{i}}-\operatorname{div} \mathbf{j}\right]=-\frac{\Pi_{i j}^{\prime}}{\tau_{2}^{(2)}} .
\end{array}\right.
$$

В этих уравнениях введены переномированные параметры:

$$
\begin{gathered}
\tau_{2}^{(2)}=\frac{\tau_{2}}{1+F_{2}}, \quad m_{0}=\frac{m}{1+F_{1}}, \\
s_{0}^{2}=\left(1+F_{1}\right) s^{2}=\frac{e^{2} 4 \pi d n_{0}}{m_{0} \kappa}, \\
\omega_{c}^{(0)}=\left(1+F_{1}\right) \omega_{c}=\frac{e B}{m_{0} c}, \\
\omega_{c}^{(2)}=\left(1+F_{2}\right) \omega_{c}=\frac{e B}{m_{0} c} \frac{1+F_{2}}{1+F_{1}} .
\end{gathered}
$$

Полученные перенормировки массы квазичастиц, скорости плазмонов и „обычной“ циклотронной частоты $\omega_{c}^{(0)}$ соответствуют приведенным в литературе [39,40]. Как известно, полученная масса $m_{0}$ есть масса электрона без учета взаимодействия [39]. Такая перенормировка массы $m$ отвечает тому факту, что частота циклотронного резонанса $\omega_{c}^{(0)}$ не меняется при учете межэлектронного взаимодействия (теорема Кона) [44].

По-видимому, неизвестным ранее результатом является следующий из формул (30) вывод о наличии двух циклотронных частот в уравнениях гидродинамики заряженной ферми-жидкости: одна из них - это „обычная“ циклотронная частота $\omega_{c}^{(0)}$, входящая в закон дисперсии магнетоплазмонов, вторая - это частота $\omega_{c}^{(2)}$, входящая в уравнение эволюции тензора вязких напряжений жидкости в магнитном поле $\sigma_{i j}^{\prime}=-\Pi_{i j}^{\prime}$. Последняя определяет частоту изученного в работе [31] вязкостного резонанса $\omega=2 \omega_{c}^{(2)}$.

Для вывода уравнения гидродинамики, содержащего только плотность потока квазичастиц $\mathbf{j}$ (уравнение Навье-Стокса заряженной ферми-жидкости), необходимо второе из уравнений (29) подставить в первое при 
фиксированной частоте течения. После преобразований, полностью аналогичных проведенным в работе [31] для случая ферми-газа, получим

$$
\begin{aligned}
-i \omega \mathbf{j}= & \frac{e n_{0}}{m_{0}} \mathbf{E}_{0}(\omega)-\left(s_{0}^{2}+c_{s}^{2}\right) \nabla \delta n+\omega_{c}^{(0)}\left[\mathbf{j} \times \mathbf{e}_{z}\right] \\
& +\eta_{x x}(\omega) \Delta \mathbf{j}+\eta_{x y}(\omega)\left[\Delta \mathbf{j} \times \mathbf{e}_{z}\right],
\end{aligned}
$$

где $\Delta-$ двумерный оператор Лапласа, $c_{s}^{2}=\left(v_{\mathrm{F}}^{2} / 2\right)\left(1+F_{0}\right)\left(1+F_{1}\right)-$ известная из литературы перенормированная скорость первого (термодинамического) звука в ферми-жидкости [39], а коэффициенты вязкости с перенормированными параметрами имеют вид

$$
\begin{aligned}
& \eta_{x x}(\omega)=\eta_{0}^{(2)} \frac{1-i \omega \tau_{2}^{(2)}}{1+\left[-\omega^{2}+\left(\omega_{c}^{(2)}\right)^{2}\right]\left(\tau_{2}^{(2)}\right)^{2}-2 i \omega \tau_{2}^{(2)}}, \\
& \eta_{x y}(\omega)=\eta_{0}^{(2)} \frac{2 \omega_{c}^{(2)} \tau_{2}^{(2)}}{1+\left[-\omega^{2}+\left(\omega_{c}^{(2)}\right)^{2}\right]\left(\tau_{2}^{(2)}\right)^{2}-2 i \omega \tau_{2}^{(2)}}
\end{aligned}
$$

Здесь $\eta_{0}^{(2)}=\left(1+F_{1}\right)\left(1+F_{2}\right) v_{\mathrm{F}}^{2} \tau_{2}^{(2)} / 4-$ перенормированный коэффициент вязкости в нулевом магнитном поле на нулевой частоте. В рассматриваемом приближении (15), когда не учитывается релаксация квазичастиц по энергии, мы получили, что коэффициент объемной вязкости равен нулю. Видно, что полученные коэффициенты вязкости имеют резонанс при $\omega=2 \omega_{c}^{(2)}$.

\section{5. Вязкоупругий переход и магнитозвуковые волны}

В пределе большой частоты, $\omega \gg 1 / \tau_{2}^{(2)}$, при нулевом магнитном поле диагональный коэффициент вязкости принимает вид

$$
\begin{gathered}
\operatorname{Im} \eta_{x x}=\left(1+F_{1}\right)\left(1+F_{2}\right) \frac{v_{\mathrm{F}}^{2}}{4} \frac{1}{\omega}, \\
\operatorname{Re} \eta_{x x} \ll \operatorname{Im} \eta_{x x} .
\end{gathered}
$$

Последнее неравенство означает малую роль диссипативных процессов по сравнению с упругими процессами (деформация сжатия и отвечающие ей возбуждения плазмонов, а также деформация сдвига). В этом пределе уравнение Навье-Стокса (31) переходит в уравнение колебаний упругой среды

$$
-\omega^{2} \varrho_{0} \mathbf{u}=K \operatorname{grad} \operatorname{div} \mathbf{u}+\mu \Delta \mathbf{u}+\mathbf{F}_{0},
$$

где $\mathbf{u}$ - вектор сдвига упругой среды, связанный с плотностью потока как $\mathbf{j}=n_{0} \partial \mathbf{u} / \partial t ; \varrho_{0}=m_{0} n_{0}-$ плотность среды; $\mathbf{F}_{0}=n_{0} \mathbf{E}_{0}-$ сила, действующая на единицу объема; $K$ - модуль всестороннего сжатия; $\mu$ - модуль сдвига. При нулевом магнитном поле упругие модули связаны с коэффициентами выведенного выше уравнения Навье-Стокса следующим образом:

$$
K=\varrho_{0}\left(s_{0}^{2}+c_{s}^{2}\right), \quad \mu=-i \omega \varrho_{0} \eta_{x x} .
$$

Таким образом, при $\omega \ll 1 / \tau_{2}^{(2)}$ эффект вязкости приводит только к процессам релаксации (например, к затуханию плазмонов), а при $\omega \gg 1 / \tau_{2}^{(2)}$ коэффициент вязкости превращается в упругую константу, сохраняя малую вещественную часть, что означает возможность существования упругих сдвиговых (поперечных) волн. Система превращается в аморфное твердое тело.

Впервые описанный вязкоупругий переход для электронной ферми-жидкости в отсутствие магнитного поля был рассмотрен в работе [38]. Полученное выражение (33) совпадает с полученным в работе [38] результатом.

В работе [32] был изучен вязкоупругий переход в двумерной электронной жидкости в магнитном поле в рамках гидродинамической модели. Было показано, что временная дисперсия коэффициентов вязкости [формулы типа (32)] приводит при $\omega \gg 1 / \tau_{2}$ к появлению поперечных магнитозвуковых волн. За счет возбуждения таких волн в отклике достаточно узкого образца на переменное электрическое поле может возникать вязкостный резонанс. В работе [32] было также установлено, что необходимым условием существования поперечных магнитозвуковых волн является сильная неидеальность электронной жидкости, которая выражается в сильном увеличении коэффициента вязкости жидкости по сравнению со случаем идеального ферми-газа:

$$
\eta_{0} \gg v_{\mathrm{F}}^{2} \tau_{2}
$$

где $\eta_{0}-$ коэффициент вязкости жидкости при $\omega, B=0$, $v_{\mathrm{F}}$ - скорость Ферми, вычисляемая из плотности жидкости по формулам для ферми-газа.

Полученный в настоящей работе в моделе сильно неидеальной ферми-жидкости результат для $\eta_{0}=\eta_{0}^{(2)}$ позволяет дать явное выражение для критерия (36):

$$
\left(1+F_{1}\right)\left(1+F_{2}\right) \gg 1 \text {. }
$$

Это неравенство выполняется при выполненности полученного выше критерия (21) прменимости гидродинамического описания волн в ферми-жидкости.

Таким образом, для возможности гидродинамического описания поперечных магнитозвуковых волн необходимо, чтобы по крайней мере параметр Ландау $F_{1}$ был велик по сравнению с единицей и параметрами Ландау старше второго.

В работе [32] был получен закон дисперсии поперечных магнитозвуковых волн в виде выражения, содержащий точно не известный макроскопический параметр $\eta_{0}$. С помощью полученных уравнений для коэффициентов вязкости (32) и формул статьи [32] можно легко получить точное выражение для дисперсии этих волн, содержащее переномированные величины $\omega_{c}^{(2)}, \eta_{0}^{(2)}, \tau_{2}^{(2)}$ (которые в свою очередь выражаются через параметры Ландау $F_{1}$ и $\left.F_{2}\right)$ :

$$
\omega_{s}(q)=\sqrt{4\left[\omega_{c}^{(2)}\right]^{2}+\frac{\eta_{0}^{(2)}}{\tau_{2}^{(2)}} q^{2}} .
$$


Отметим, что величина $\sqrt{\eta_{0}^{(2)} / \tau_{2}^{(2)}}$ обозначалась в работе как перенормированная „вязкостная“ скорость Ферми $v_{F}^{\eta}$, для которой выполнено $v_{F}^{(\eta)} \gg v_{F}$. При больших значениях $F_{1}$ и $F_{2}$ получаем

$$
\omega_{s}(q)=\frac{F_{2}}{F_{1}} \sqrt{4\left[\omega_{c}^{(0)}\right]^{2}+\frac{F_{1}^{3}}{F_{2}} \frac{v_{\mathrm{F}}^{2}}{4} q^{2}}
$$

Разумно ожидать, что гармоники функции Ландау спадают с ростом их номера уже в области малых номеров $m=0,1,2$, как это обычно бывает для регулярных периодических функций. Поэтому, по-видимому, типично, что $F_{2} / F_{1}<1$. Это означает, что частота вязкостного резонанса $\omega=2 \omega_{c}^{(2)}$ с расположена в области более низких частот $\omega$, чем удвоенная частота циклотронного резонанса $2 \omega_{c}^{(0)}$.

Из формулы (39) видно, что при $\omega \sim \omega_{c}$ длина волны поперечного нулевого звука есть величина порядка $l_{s} \sim \sqrt{F_{1} F_{2}} v_{\mathrm{F}} / \omega$. Эта величина много больше расстояния $l_{\omega} \sim v_{\mathrm{F}} / \omega$, проходимого квазичастицей за один период изменения внешнего поля: $l_{\omega} \ll l_{s}$. Таким образом, мы получаем, что сформулированный в начале статьи наглядный критерий применимости гидродинамического приближения действительно выполнен.

Из уравнения непрерывности [первое уравнение в системе (28)] и уравнения Навье-Стокса (31) легко получить, что спектр продольных волн, связанных с возмущением плотности, в сильно неидеальной фермижидкости будет определяться формулой

$$
w_{s}(q)=\sqrt{\left[\omega_{c}^{(0)}\right]^{2}+\left(s_{0}^{2}+c_{s}^{2}\right) q^{2}} .
$$

Отсюда и из (30) видно, что при достаточно больших значениях параметров $F_{0}$ и $F_{1}$ скорость плазмонов $s_{0}$ может сравниться с перенормированной скоростью звука $c_{s}$. Следовательно, такие волны не будут иметь чисто электрическую природу, как магнетоплазмоны, а будут одновременно связаны как с возбуждением объемного заряда и соответсвующего внутреннего электрического поля, так и с возникновением неоднородного давления.

\section{6. Заключение}

В работе показано, что динамика возбуждений сильно неидеальной двумерной электронной ферми-жидкости описывается уравнениями гидродинамики, учитывающими временную дисперсию вязкости и возникновение объемного заряда. Продемонстрировано, что при высоких частотах поведение системы становится похожим на динамику аморфного твердого тела, а именно становится возможным возбуждение поперечных магнитозвуковых волн (поперечного нулевого звука). Показано, что в заряженной ферми-жидкости перенормируются не только эффективная масса квазичастиц и амплитуда коэффициентов вязкости, но также время релаксации и циклотронная частота, входящие в коэффициенты вязкости. В результате такая „Вязкостная“ циклотронная частота становится отличной от обычной циклотронной частоты, определяющей циклотронный резонанс. Рассчитаны законы дисперсии поперечных и продольных магнетозвуковых волн.

\section{Благодарности}

Автор благодарит М.И. Дьяконова за советы, поддержку и многочисленные обсуждения теоретических вопросов и экспериментальных данных, которые легли в основу этой работы; а также Н.С. Аверкиева, А.П. Алексееву, Е.Г. Алексееву, И.П. Алексееву, С.А. Балашева, М.М. Глазова, Л.Е. Голуба, И.В. Горного, А.П. Дмитриева, С.А. Левшакова, Р. Меснера, М.О. Нестоклона, А.Н. Поддубного, С.М. Постолова, Д.С. Свинкина, И. Содемана, П. Суровку, С.А. Тарасенко, Б.И. Шкловского и Д.Г. Яковлева за поддержку, советы и полезные обсуждения.

\section{Финансирование работы}

Исследование выполнено за счет гранта Российского научного фонда (проект № 18-72-10111).

\section{Конфликт интересов}

Авторы заявляют об отсутствии конфликта интересов.

\section{Список литературы}

[1] Р.Н. Гуржи, УФН, 94, 689 (1968).

[2] В.Л. Гуревич. Кинетика фононных систем (М., Наука, 1980).

[3] В.Л. Гуревич, Б.И. Шкловский. ФТТ, 8, 3050 (1956).

[4] Kh. Nil'sen, B.I. Shklovskii. Sov. Phys. Solid State, 10, 2857 (1969).

[5] A.T. Hatke, M.A. Zudov, J.L. Reno, L.N. Pfeiffer, K.W. West. Phys. Rev. B, 85, 081304 (2012).

[6] R.G. Mani, A. Kriisa, W. Wegscheider. Sci. Rep., 3, 2747 (2013).

[7] L. Bockhorn, P. Barthold, D. Schuh, W. Wegscheider, R.J. Haug, Phys. Rev. B, 83, 113301 (2011).

[8] Q. Shi, P.D. Martin, Q.A. Ebner, M.A. Zudov, L.N. Pfeiffer, K.W. West. Phys. Rev. B, 89, 201301 (2014).

[9] G.M. Gusev, A.D. Levin, E.V. Levinson, A.K. Bakarov. AIP Adv., 8, 025318 (2018).

[10] A.D. Levin, G.M. Gusev, E.V. Levinson, Z.D. Kvon, A.K. Bakarov. Phys. Rev. B, 97, 245308 (2018).

[11] P.J.W. Moll, P. Kushwaha, N. Nandi, B. Schmidt, A.P. Mackenzie. Science, 351, 1061 (2016).

[12] J. Gooth, F. Menges, C. Shekhar, V. Suess, N. Kumar, Y. Sun, U. Drechsler, R. Zierold, C. Felser, B. Gotsmann. Nature Commun., 9, 4093 (2018).

[13] D.A. Bandurin, I. Torre, R. Krishna Kumar, M. Ben Shalom, A. Tomadin, A. Principi, G.H. Auton, E. Khestanova, K.S. NovoseIov, I.V. Grigorieva, L.A. Ponomarenko, A.K. Geim, M. Polini. Science, 351, 1055 (2016). 
[14] R. Krishna Kumar, D.A. Bandurin, F.M.D. Pellegrino, Y. Cao, A. Principi, H. Guo, G.H. Auton, M. Ben Shalom, L.A. Ponomarenko, G. Falkovich, K. Watanabe, T. Taniguchi, I.V. Grigorieva, L.S. Levitov, M.Polini, A.K. Geim. Nature Phys., 13, 1182 (2017).

[15] A.I. Berdyugin, S.G. Xu, F.M.D. Pellegrino, R. Krishna Kumar, A. Principi, I. Torre, M. Ben Shalom, T. Taniguchi, K. Watanabe, I.V. Grigorieva, M. Polini, A.K. Geim, D.A. Bandurin. Science, 364, 162 (2019).

[16] M. Hruska, B. Spivak. Phys. Rev. B, 65, 033315 (2002).

[17] A.V. Andreev, S.A. Kivelson, B. Spivak. Phys. Rev. Lett., 106, 256804 (2011).

[18] P.S. Alekseev. Phys. Rev. Lett., 117, 166601 (2016).

[19] L. Levitov, G. Falkovich. Nature Phys., 12, 672 (2016).

[20] A. Lucas. Phys. Rev. B, 95, 115425 (2017).

[21] F.M.D. Pellegrino, I. Torre, M. Polini. Phys. Rev. B, 96, 195401 (2017).

[22] P.S. Alekseev, A.P. Dmitriev, I.V. Gornyi, V.Y. Kachorovskii, M.A. Semina. Semiconductors 51, 766 (2017).

[23] P.S. Alekseev, A.P. Dmitriev, I.V. Gornyi, V.Yu. Kachorovskii, B.N. Narozhny, M. Titov. Phys. Rev. B, 97, 085109 (2018).

[24] P.S. Alekseev, A.P. Dmitriev, I.V. Gornyi, V.Yu. Kachorovskii, B.N. Narozhny, M. Titov. Phys. Rev. B, 98, 125111 (2018).

[25] P.S. Alekseev, M.A. Semina. Phys. Rev. B, 98, 165412 (2018).

[26] O. Kashuba, B. Trauzettel, L.W. Molenkamp. Phys. Rev. B, 97, 205129 (2018).

[27] R. Moessner, P. Surowka, P. Witkowski. Phys. Rev. B, 97, 161112 (2018)

[28] M. Semenyakin, G. Falkovich. Phys. Rev. B, 97, 085127 (2018).

[29] R. Cohen, M. Goldstein. Phys. Rev. B, 98, 235103 (2018).

[30] J.Y. Khoo, I.S. Villadiego. Phys. Rev. B 99, 075434 (2019).

[31] P.S. Alekseev. Phys. Rev. B, 98, 165440 (2018).

[32] P.S. Alekseev, A.P. Alekseeva. arXiv:1810.10241 (2018).

[33] Y. Dai, R.R. Du, L.N. Pfeiffer, K.W. West. Phys. Rev. Lett., 105, 246802 (2010).

[34] A.T. Hatke, M.A. Zudov, L.N. Pfeiffer, K.W. West. Phys. Rev. B, 83, 121301 (2011).

[35] M. Bialek, J. Lusakowski, M. Czapkiewicz, J. Wrobel, V. Umansky. Phys. Rev B, 91, 045437 (2015).

[36] И.В. Кукушкин, В.А. Волков, Двумерная электронная жидкость в сильном магнитном поле (М., Физматкнига, 2016).

[37] A.V. Shchepetilnikov, D.D. Frolov, Yu.A. Nefyodov, I.V. Kukushkin, S. Schmult. Phys. Rev. B, 95, 161305 (2017).

[38] S. Conti, G. Vignale. Phys. Rev. B, 60, 7966 (1999).

[39] Е.М. Лифшиц, Л.П. Питаевский. Статистическая физи$\kappa a$ (М., Физматлит, 2004) ч. 2.

[40] Д. Пайнс, Ф. Нозьер. Теория квантовых жмидкостей (М., Мир, 1967).

[41] Е.М. Лифшиц, Л.П. Питаевский. Физическал кинетика (М., Наука, 1979).

[42] I.M. Khalatnikov, A.A. Abrikosov. Sov. Phys. JETP, 6, 84 (1958).

[43] J. Sykes, G.A. Brooker. Ann. Phys., 56, 1 (1970).

[44] W. Kohn. Phys. Rev. 123, 1242 (1961).

\section{Magnetosonic waves in a two-dimensional electron Fermi liquid}

\section{P.S. Alekseev}

loffe Institute, 194021 St. Petersburg, Russia

Abstract The properties of highly viscous fluids at high frequencies become similar to the properties of amorphous solids. In particular, it becomes possible to propagate not only longitudinal sound waves (plasmons for the case of an electron fluid), but also transverse sound waves associated with shear deformations. In this work, transverse sound waves at high frequencies in a twodimensional electron liquid in a magnetic field are studied. The consideration was carried out in the framework of the Landau Fermi-liquid model. It is shown that for a sufficiently large interaction between quasiparticles, the dynamics of excitations of a Fermi liquid is described by the equations of hydrodynamics. The Navier-Stokes equation and expressions for high-frequency shear viscosity coefficients are derived. Based on the equations obtained, the dispersion laws are calculated for transverse and longitudinal magnetosonic waves. It is shown that the cyclotron frequency, which enters in the viscosity coefficients and the dispersion law of transverse magnetosonic waves, is renormalized and typically becomes less than the usual cyclotron frequency, which determines the cyclotron resonance. The latter fact was apparently observed in the photoresistance of highly mobile GaAs quantum wells, in which two-dimensional electrons form a viscous fluid.

Редактор А.Н. Смирнов 